\title{
Follicular Dendritic Cell Sarcoma of the Tonsil: A Multimodality Approach
}

\author{
Rich Ericson C. King ${ }^{\mathrm{a}, \mathrm{e}}$, Andrea R. Villaruel ${ }^{\mathrm{b}}$, Jose Pedrito M. Magno ${ }^{\mathrm{c}}$,

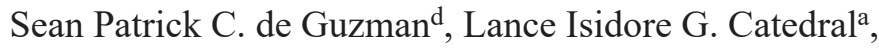 \\ Karen Anjela M. Mondragon ${ }^{\mathrm{a}}$, Frederic Ivan L. Tinga, \\ Rogelio N. Velasco ${ }^{a}$, Gracieux Y. Fernando ${ }^{a}$
}

\begin{abstract}
Follicular dendritic cell sarcoma (FDCS) accounts for $<0.4 \%$ of soft tissue sarcomas. Only 35 cases of tonsillar FDCS have been reported, and majority had localized presentation. We present a case of FDCS of the tonsil, wherein a well-coordinated trimodality approach provided good disease control in advanced disease. A 53-year-old man presented with a painless and enlarging neck mass of 11-month duration, with no other symptoms. Close examination revealed a $10 \times 5$ $\mathrm{cm}$ mass at the left carotid triangle, and a $3.2 \times 2.2 \mathrm{~cm}$ mass at the left tonsillar fossa. Imaging revealed the tumor to be unresectable due to its attachment to the great vessels. There were no distant metastases. Biopsy and immunohistochemistry were initially deemed consistent with an undifferentiated sarcoma. Palliative chemotherapy was given using single agent doxorubicin and subsequent dacarbazine, resulting in partial response and stable disease, respectively. Pathological re-evaluation was pursued because of the uncharacteristic slow progression of the tumor, revealing diffuse positivity for CD21 and negative for CD1A and CD34, consistent with FDCS. The patient underwent three cycles of gemcitabine plus docetaxel resulting in 50\% regression. This allowed dissection of level IB-V lymph nodes and subsequent radiotherapy for the neck and tonsillar mass, with weekly gemcitabine as a radiosensitizer. Evaluation 8 months post-treatment showed no signs of disease progression. Treatment-related complications included radiation dermatitis and swallowing dysfunction, which both resolved on follow-up. This case highlights the multidisciplinary management of a rare type of sarcoma in an uncommon ana-
\end{abstract}

Manuscript submitted July 24, 2020, accepted July 31, 2020

Published online August 28, 2020

aDivision of Medical Oncology, Philippine General Hospital, Taft Avenue, Manila, Philippines

bDepartment of Laboratories, Philippine General Hospital, Taft Avenue, Manila, Philippines

'Department of Otorhinolaryngology, Philippine General Hospital, Taft Avenue, Manila, Philippines

dDepartment of Radiology, Philippine General Hospital, Taft Avenue, Manila, Philippines

${ }^{e}$ Corresponding Author: Rich Ericson C. King, Division of Medical Oncology, Philippine General Hospital, Taft Avenue, Manila 1000, Philippines.

Email: richericson.king@gmail.com

doi: https://doi.org/10.14740/jmc3551 tomic location. Precise pathologic diagnosis is important in soft tissue sarcoma because of its therapeutic implications. For FDCS, effective response may still be achieved in the third-line setting.

Keywords: Soft tissue sarcoma; Follicular dendritic cell sarcoma; Head and neck; Malignancy; Sarcoma

\section{Introduction}

Follicular dendritic cell sarcoma (FDCS) is a rare neoplasm of follicular dendritic cell differentiation. Because of its rarity, a high index of suspicion is necessary for timely diagnosis. Because presentation is often localized, there is still no standard treatment for advanced disease.

We report a case of FDCS of the tonsil (an uncommon site of involvement) with multiple cervical lymph node metastases. Accurate pathologic diagnosis was crucial in the choice of systemic therapy, which afforded significant clinical response even in the third-line setting. In addition, close collaboration between the head and neck surgeons, medical oncologists, radiation oncologists and pathologists was instrumental in achieving good disease control.

\section{Case Report}

A 53-year-old male electrical technician presented with a painless enlarging neck mass of 11-month duration. He denied having any constitutional symptoms and only complained of dysphagia. He was a 40-pack-year smoker and only occasionally drank alcoholic beverages. He had a previous diagnosis of subclinical hyperthyroidism, for which he had been under monitoring. Pertinent family and surgical history were negative.

\section{Initial evaluation}

A contrast-enhanced computed tomography (CT) scan of the neck showed a $10 \times 5 \mathrm{~cm}$ mass at the left carotid triangle, and 


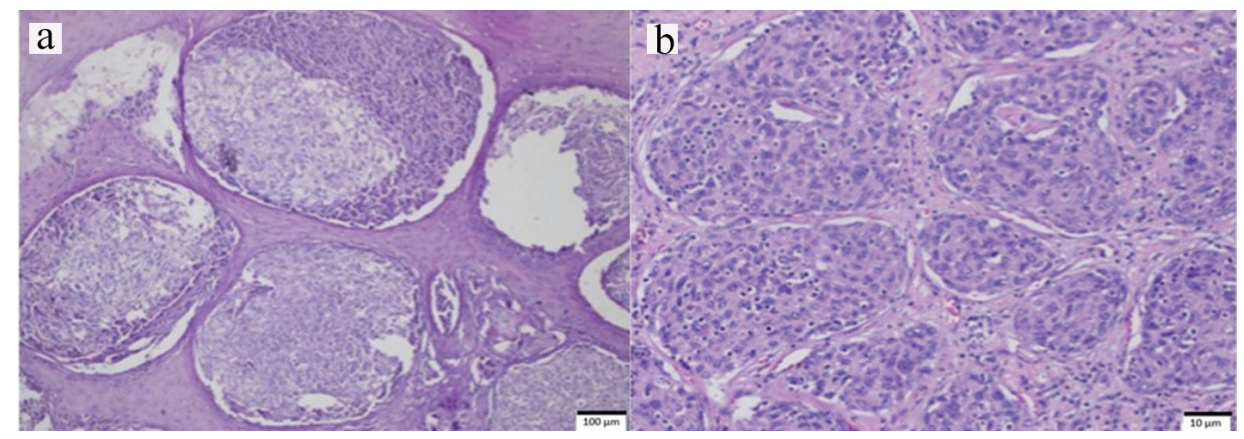

Figure 1. Punch biopsy specimen of the tonsillar mass on hematoxylin and eosin staining. (a) Scanning $1 \times$ objective of the tumor shows cells that are arranged in nests and vague nodules. In some areas, they are separated by septa of stratified squamous epithelium, which is the normal lining of the tonsil. The tumor cells are also closely admixed with a population of benign lymphocytes. (b) On $10 \times$ objective, the individual cells are ovoid to spindle with vesicular nuclei. Some have indistinct cell borders, exhibiting a slight storiform or whorled pattern.

a $3.2 \times 2.2 \mathrm{~cm}$ mass at the left tonsillar fossa causing compression deformity to the base of the tongue and soft palate. Punch biopsy of the tonsillar mass revealed an undifferentiated malignancy. Immunohistochemistry was negative for cytokeratin, leucocyte common antigen (LCA), epithelial membrane antigen (EMA), synaptophysin, chromogranin and 34ßE12, diffusely positive for vimentin, and focally positive for S-100, favoring malignant melanoma. However, subsequent immunohistochemical staining with HMB-45 and melan-A was negative, leading to a classification of undifferentiated sarcoma.

Chest and abdominal CT scan were negative for distant metastases. His complete blood count as well as kidney, liver and thyroid function tests was within normal limits. The case was discussed in the hospital head and neck multidisciplinary conference consisting of head and neck surgeons, medical oncologists, radiation oncologists and pathologists. The tumor was deemed unresectable due to its attachment to the great vessels; hence, a sequential chemoradiation approach was planned.

\section{Initial treatment}

He underwent chemotherapy with six cycles of doxorubicin $\left(60 \mathrm{mg} / \mathrm{m}^{2}\right)$, resulting in $80 \%$ tumor regression and resolution of his dysphagia. Unfortunately, he was lost to follow-up and was unable to pursue radiotherapy. Eight months later, he returned with re-enlargement of the mass to $6 \times 5 \mathrm{~cm}$. He remained free of distant metastases and underwent two cycles of dacarbazine $\left(250 \mathrm{mg} / \mathrm{m}^{2}\right.$, days $\left.1-5\right)$, with only stable disease as response.

\section{Approach to diagnosis}

The relatively long-time interval to progression in the setting of an undifferentiated histology prompted pathological reevaluation of the tumor. The tonsillar location of the tumor and its close association with lymphoid tissue suggested a hematolymphoid origin. In particular, suggestive morphologic features (Fig. 1) and the lack of staining for LCA favored a diagnosis of a histiocytic or a dendritic cell tumor rather than a lymphoma. Staining for CD34 was performed to rule out a myeloid tumor, while staining for CD21 and CD1A was used to support a diagnosis of FDCS or Langerhans cell histiocytosis, respectively.

Immunohistochemistry was performed on the original specimen revealing that CD1A and CD34 were both negative. On the other hand, the tumor exhibited diffuse staining for CD21, confirming the diagnosis of FDCS.

\section{Subsequent treatment}

The patient underwent three cycles of gemcitabine $(1,000 \mathrm{mg} /$ $\mathrm{m}^{2}$, days 1,8$)$ plus docetaxel $\left(75 \mathrm{mg} / \mathrm{m}^{2}\right.$, day 1$)$, resulting in $50 \%$ regression as best response (Fig. 2). This allowed dissection of level IB-V cervical lymph nodes, which found disease in 10/23 nodes. He subsequently underwent adjuvant radiotherapy to the neck (60 Gy) and primary radiotherapy to the tonsillar mass (70 Gy), with weekly gemcitabine $\left(400 \mathrm{mg} / \mathrm{m}^{2}\right)$ as a radiosensitizer.

\section{Treatment outcome and follow-up}

Treatment-related toxicities included radiation dermatitis to the involved neck and swallowing dysfunction for which he was enrolled in a swallowing rehabilitation program. At 8 months post-treatment, he has attained significant improvement in swallowing function without any sign of disease progression.

\section{Discussion}

\section{FDCS}

FDCS comprises $<0.4 \%$ of all soft tissue sarcomas. They exhibit morphologic and immunophenotypic features of follicular dendritic cells, antigen-presenting cells that reside in pri- 

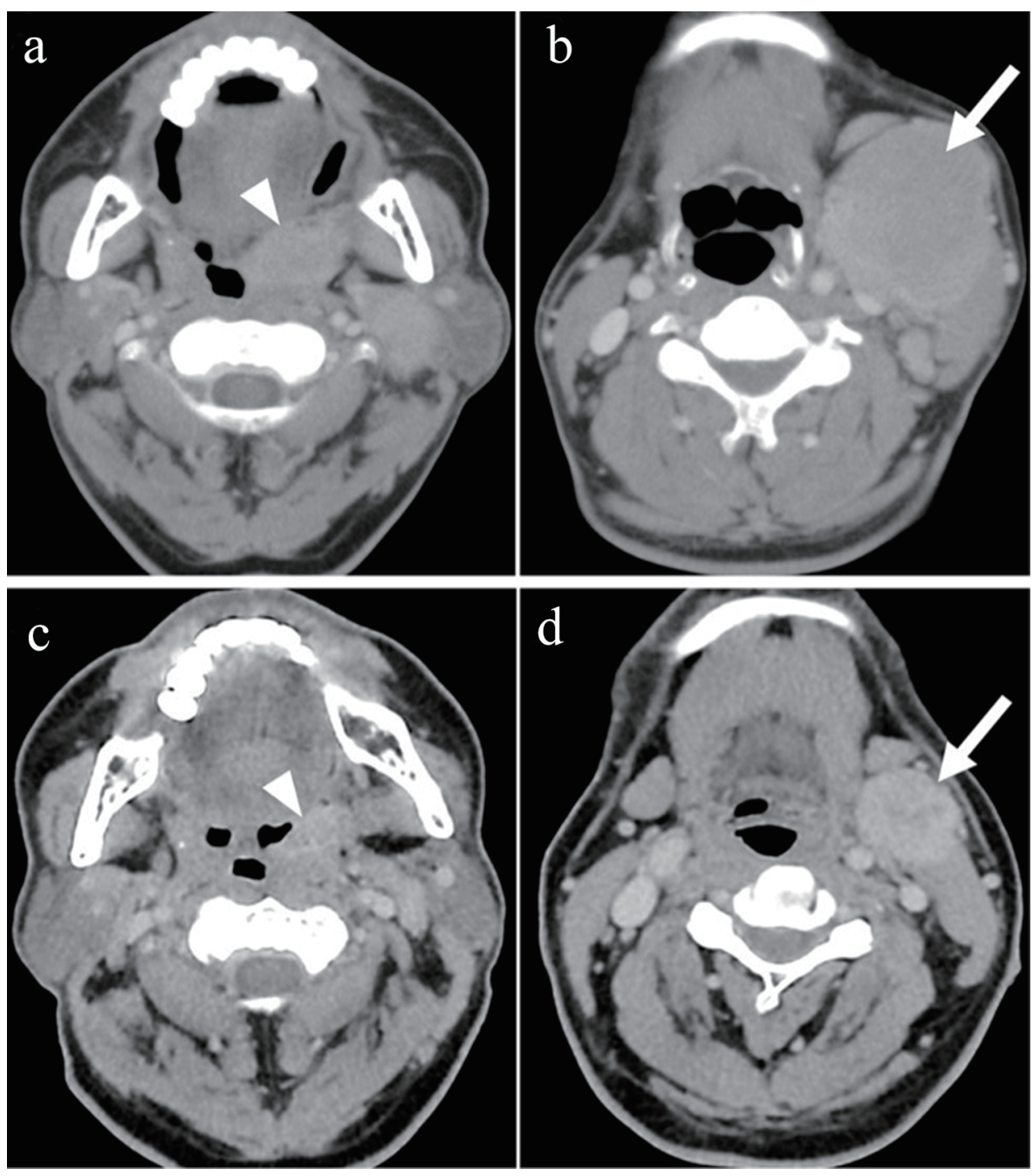

Figure 2. Pre-treatment axial contrast-enhanced CT scan images ( $a$ and b) show an enhancing mass lesion in the left faucial tonsil (arrowhead) with large submandibular nodal metastasis (arrow) which decreased in size on evaluation after three cycles of gemcitabine and docetaxel (c and d). CT: computed tomography.

mary and secondary B-cell follicles. Under the World Health Organization (WHO) classification of tumors of hematopoietic and lymphoid tissues, FDCS is classified under histiocytic and dendritic cell neoplasms together with histiocytic sarcoma, interdigitating dendritic cell sarcoma, Langerhans cell histiocytosis and Langerhans cell sarcoma [1].

The cause is unknown for most patients with the disease, although a small minority may present as an inflammatory pseudotumor-like variant that has been linked with EpsteinBarr virus (EBV) infection. This variant tends to occur in women with an intraabdominal presentation, is associated with constitutional symptoms, and is observed to have a more indolent behavior. Another 5-10\% may coexist or be preceded by a diagnosis of Castleman's disease, suggesting a link with this benign lymphoproliferative disorder. Autoimmune conditions, particularly paraneoplastic pemphigus and myasthenia gravis, have been observed as well [2]. Our patient had a history of subclinical hyperthyroidism, although this has normalized at the time of evaluation and no workup for autoimmune etiolo- gies was performed.

\section{Epidemiology}

The mean age of diagnosis of FDCS is 49 years (ranging from 9 to 90 years), with men and women being equally affected. Extranodal involvement comprises $65 \%$ of reported cases, while nodal involvement is seen in $25 \%$, and a combination of both nodal and extranodal in the remaining 10\% [3]. Eighty-five percent of cases present with early disease, while distant metastases are encountered in only eight percent [2]. The liver and spleen are the most common extranodal sites involved, while only 35 cases of tonsillar occurrence have been reported in literature [4].

\section{Pathologic features}

Morphologic features of FDCS consist of spindled to ovoid 
cells forming fascicles, whorls, sheets or vague nodules, often with intermixed small lymphocytes. Misdiagnosis is common because as a rare tumor, it is often not considered in the differential diagnosis of spindle cell neoplasms. Depending on the location of the tumor, differential diagnoses may include inflammatory myofibroblastic tumor, undifferentiated carcinoma, undifferentiated pleomorphic sarcoma, lymphoma, schwannoma, gastrointestinal stromal tumor, and other histiocytic or dendritic cell tumors [5].

Staining for follicular dendritic cell markers such as CD21, CD35, CD23, KiM4p or CAN confirms the diagnosis [1]. FDCS can also have variable staining for vimentin, CD68 and S100, but is consistently negative for cytokeratins, CD31, CD34 and CD1a [5]. For the present case, immunohistochemical expression of vimentin and S100 led to an initial consideration of malignant melanoma, especially since it can also present with a whorling pattern with intermixed lymphocytes [1]. However, confirmatory stains melan-A and HMB-45 were negative. The correct diagnosis was established months later when pathological re-evaluation raised the suspicion for a dendritic cell neoplasm based on the tumor location and its morphologic features.

\section{Management of localized disease}

Surgical resection is the mainstay of treatment for localized FDCS. A review of 147 patients with localized disease showed that patients who underwent surgery had a significantly better survival compared to those who had other treatment modalities [4]. The benefit of adjuvant chemotherapy or radiotherapy is much less clear. Analyses of retrospective data regarding adjuvant therapy for localized FDCS have failed to demonstrate benefit in terms of recurrence rates, metastasis or survival [68].

\section{Systemic therapy for advanced disease}

For locally advanced or metastatic disease, chemotherapy with or without radiotherapy can achieve effective disease control. Reported cases of advanced FDCS with available treatment and outcome information are summarized in Table 1 [9-32]. Out of 34 cases (including our patient), cyclophosphamide, doxorubicin, vincristine and prednisone (CHOP) chemotherapy was used as the first-line regimen in 53\%, resulting in complete response in four and partial response in 10 patients. Unfortunately, our patient has already been given a total dose of $360 \mathrm{mg} / \mathrm{m}^{2}$ of doxorubicin before the diagnosis of FDCS was established.

Other regimens that were used include those that are used for lymphomas (ifosfamide, carboplatin and etoposide (ICE) [11, 13, 24], dexamethasone, cytarabine and cisplatin (DHAP) $[9,13]$, etoposide, prednisone, vincristine, cyclophosphamide and doxorubicin (EPOCH) [10], etoposide, methylprednisolone, cytarabine and cisplatin (ESHAP) [13, 27, 30, 31], ifosfamide, gemcitabine, vinorelbine and prednisolone (IGEV) [19]) and soft tissue sarcomas (doxorubicin, ifosfamide and mesna (AIM) [14, 20, 21, 31], ifosfamide and etoposide (IE) [13], gemcitabine and docetaxel (Gem/Doce) [13, 22, 23, 32]). Notably, all three sarcoma regimens have been demonstrated to induce complete response, with the combination of gemcitabine and docetaxel resulting in complete response in the third-line setting in one case [13]. Thus, this combination was the one chosen for our patient, which resulted in a 50\% regression of the tumor as a third-line treatment.

Some oncologists favor treating FDCS with a sarcoma regimen such as Gem/Doce over CHOP. This is based on the observation that follicular dendritic cell precursors are of vascular stromal rather than of lymphoid origin. Furthermore, it is theorized that the response of FDCS to CHOP may involve the depletion of B lymphocytes that release growth factors to follicular dendritic cells, rather than directly causing cytotoxicity to cancer cells. Its mesenchymal origin and the behavior of FDCS being more similar to an intermediate grade soft tissue sarcoma rather than a lymphoma give support to this perspective [22]. At present, comparative studies investigating the response of FDCS to different chemotherapy regimens do not exist.

Of interest, one patient was found by immunohistochemistry to have a tumor that expressed CD117, wherein treatment with a combination of gemcitabine and cisplatin for eight cycles together with imatinib resulted in a complete response [14]. In another patient, detection of a BRCA2 mutation allowed treatment with the combination of carboplatin and veliparib to achieve disease stability [29]. These examples highlight the fact that there is much more to be learned about the biology and optimal treatment of this rare malignancy.

Remarkably, there have been few cases wherein significant clinical response was still achieved after multiple lines of treatment. For example, a 44-year-old woman with metastatic FDCS had complete response with CHOP, and subsequently with ESHAP, and lastly with the combination of gemcitabine, vinorelbine and dexamethasone after presenting with multiple recurrences. At 29 months after completion of treatment, she remained free of any evidence of disease [27]. Another patient continued to achieve effective disease control after six lines of different treatment regimens [30].

\section{Radiotherapy for advanced disease}

Radiotherapy has been shown to be beneficial in the management of advanced FDCS, especially in cases wherein the site of recurrence is localized. In this scenario, the patient may be spared from chemotherapy and still achieve a long disease-free interval, and possibly have a chance for a cure. The three reported patients $[21,28,32]$ who had radiotherapy of localized recurrence remained free of metastatic progression at the time of last follow-up, which extended to as long as 6 years posttreatment for one patient [21].

For this reason, we opted to proceed with primary radiotherapy of the tonsillar mass for our patient, especially since the cervical node metastases have been successfully controlled by surgery. Adjuvant radiotherapy to the dissected neck, with gemcitabine as a radiosensitizer, was done because the size and 
Table 1. Summary of Reported Clinical Data on 34 Cases of Advanced FDCS Treated With Various Chemotherapeutic Regimens

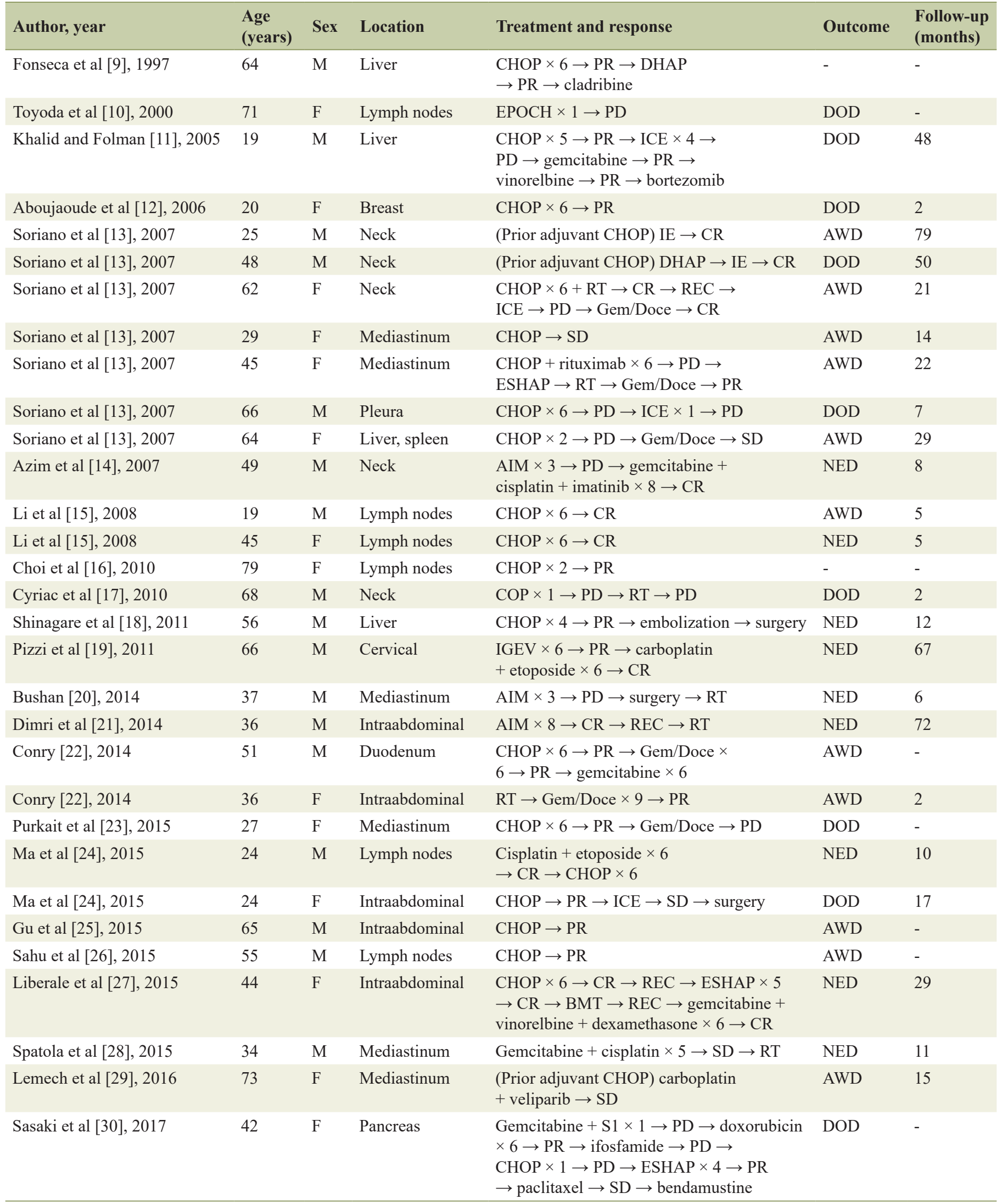


Table 1. Summary of Reported Clinical Data on 34 Cases of Advanced FDCS Treated With Various Chemotherapeutic Regimens - (continued)

\begin{tabular}{lllllll}
\hline Author, year & $\begin{array}{l}\text { Age } \\
\text { (years) }\end{array}$ & Sex & Location & Treatment and response & Outcome & $\begin{array}{l}\text { Follow-up } \\
\text { (months) }\end{array}$ \\
\hline Bouriga et al [31], 2018 & 48 & F & Retroperitoneum & AIM $\times 3 \rightarrow$ PD $\rightarrow$ ESHAP $\times 3 \rightarrow$ SD & AWD & 14 \\
Chen et al [32], 2019 & 66 & F & Liver & Gem/Doce $\times 8 \rightarrow$ PR $\rightarrow$ RT & AWD & 12 \\
Present case & 53 & M & Tonsil & $\begin{array}{l}\text { Doxorubicin } \times 6 \rightarrow \text { PR } \rightarrow \text { dacarbazine } \\
\times 2 \rightarrow \text { SD } \rightarrow \text { Gem/Doce } \rightarrow \text { PR }\end{array}$ & AWD & 8 \\
\end{tabular}

AIM: doxorubicin + ifosfamide + mesna; BMT: bone marrow transplantation; CHOP: cyclophosphamide + doxorubicin + vincristine + prednisone; COP: cyclophosphamide + vincristine + prednisone; DHAP: dexamethasone + cytarabine + cisplatin; EPOCH: etoposide + prednisone + vincristine + cyclophosphamide + doxorubicin; ESHAP: etoposide + methylprednisolone + cytarabine + cisplatin; Gem/Doce: gemcitabine + docetaxel; ICE: ifosfamide + carboplatin + etoposide; IE: ifosfamide + etoposide; IGEV: ifosfamide + gemcitabine + vinorelbine + prednisolone; RT: radiotherapy; CR: complete response; PR: partial response; SD: stable disease; PD: progressive disease; REC: recurrence; AWD: alive with disease; DOD: died of disease; NED: no evidence of disease.

number of affected lymph nodes were considered high risk for local recurrence. While the treatment goal remains to be palliative in nature, it was judged by the multidisciplinary team that radiotherapy would confer benefit to the patient because of the high-risk features mentioned.

\section{Prognosis}

FDCS is now considered by some as an intermediate-grade soft tissue sarcoma, given its tendency for recurrence and distant metastasis. Tumor size of $\geq 5 \mathrm{~cm}$ and high histologic grade have been found to be associated with a lower disease-free and overall survival [33]. In a review of 97 cases of FDCS of the head and neck, the incidence of locoregional and distant recurrence was $38 \%$ and $7 \%$, respectively. In this population of patients, locoregional recurrence rate was found to be significantly lower among patients who underwent neck dissection and among those who received adjuvant radiotherapy [34].

Survival is worse among patients with metastatic or recurrent disease (median of 2.69 years) compared to those with localized disease (median of 9.8 years) [8]. Nevertheless, review of reported cases shows that it is sensitive to available chemotherapy regimens, wherein complete response can be achieved even in the metastatic setting. Our patient responded favorably to a combination of chemotherapy, surgery and radiotherapy, although long-term follow-up is not yet available at the time of writing of this report.

\section{Learning points}

For the case presented, pathologic diagnosis was crucial in selecting effective therapy and in determining prognosis. Even for advanced FDCS, response to systemic treatment may still be achieved in the third-line setting, and this can be achieved with regimens used for lymphoma or soft tissue sarcoma.

\section{Conclusions}

Tonsillar FDCS represents an uncommon location for a rare type of sarcoma, with only 35 such cases reported in literature. Majority of cases present as localized disease where surgical resection is the mainstay of treatment. While no standard of treatment exists for advanced disease, we demonstrate in this case that good disease control may still be achieved through a well-coordinated approach consisting systemic therapy, surgery and radiotherapy.

\section{Acknowledgments}

The authors would like to acknowledge the University of the Philippines, Manila Research and Ethics Board and the Department of Medicine Clinical Research Division for administrative assistance that was provided in the preparation of this manuscript.

\section{Financial Disclosure}

None to declare.

\section{Conflict of Interest}

None to declare.

\section{Informed Consent}

The authors attest that informed consent was obtained from the patient prior to the writing of this manuscript.

\section{Author Contributions}

All authors contributed to the drafting and subsequent revisions of the manuscript. Final approval was obtained from all authors. RECK contributed to case presentation and discussion of rationale for therapeutic decisions; ARV discussed pathological aspect of the case; JPMM discussed surgical aspect of 
the case; SPCDG discussed the radiation oncology aspect of the case; LIGC discussed oncologic management of advanced disease; KAMM discussed oncologic management of localized disease; FILT discussed published cases of advanced FDCS treated with chemotherapy; RNV discussed published cases of advanced FDCS treated with chemotherapy; GYF discussed multidisciplinary management of FDCS.

\section{Data Availability}

The authors declare that data supporting the findings of this study are available within the article.

\section{References}

1. Swerdlow SH, Campo E, Harris NL, Jaffe ES, Pileri SA, Stein H, Thiele J. WHO classification of tumours of haematopoietic and lymphoid tissues [Internet]. [cited 2020 Jul 24]. Available from: https://publications.iarc. fr/Book-And-Report-Series/Who-Classification-Of-Tumours/WHO-Classification-Of-Tumours-Of-Haematopoietic-And-Lymphoid-Tissues-2017.

2. Saygin C, Uzunaslan D, Ozguroglu M, Senocak M, Tuzuner N. Dendritic cell sarcoma: a pooled analysis including 462 cases with presentation of our case series. Crit Rev Oncol Hematol. 2013;88(2):253-271.

3. Facchetti F, Lorenzi L. Follicular dendritic cells and related sarcoma. Semin Diagn Pathol. 2016;33(5):262-276.

4. Wu B, Lim CM, Petersson F. Primary tonsillar epithelioid follicular dendritic cell sarcoma: report of a rare case mimicking undifferentiated carcinoma and a brief review of the literature. Head Neck Pathol. 2019;13(4):606-612.

5. Chen T, Gopal P. Follicular dendritic cell sarcoma. Arch Pathol Lab Med. 2017;141(4):596-599.

6. Li J, Zhou ML, Zhou SH. Clinical and pathological features of head and neck follicular dendritic cell sarcoma. Hematology. 2015;20(10):571-583.

7. Hu T, Wang X, Yu C, Yan J, Zhang X, Li L, Li X, et al. Follicular dendritic cell sarcoma of the pharyngeal region. Oncol Lett. 2013;5(5):1467-1476.

8. Gounder M, Desai V, Kuk D, Agaram N, Arcila M, Durham B, Keohan ML, et al. Impact of surgery, radiation and systemic therapy on the outcomes of patients with dendritic cell and histiocytic sarcomas. Eur J Cancer. 2015;51(16):2413-2422.

9. Fonseca R, Tefferi A, Strickler JG. Follicular dendritic cell sarcoma mimicking diffuse large cell lymphoma: a case report. Am J Hematol. 1997;55(3):148-155.

10. Toyoda K, Taniguchi J, Kikawa K, Uike N, Haraoka S, Ooshima K, Kikuchi M, et al. Follicular dendritic cell sarcoma: ultrastructural and immunohistochemical studies. Intern Med. 2000;39(11):950-955.

11. Khalid T, Folman R. Symptoms in cancer patients and an unusual tumor: Case 3. Follicular dendritic cell sarcoma. J Clin Oncol. 2005;23(36):9425-9426.

12. Aboujaoude R, Alvarez JR, Alvarez M, Al-Khan A. Follicular dendritic cell sarcoma in pregnancy: case report and review of the literature. Am J Perinatol. 2006;23(8):459461.

13. Soriano AO, Thompson MA, Admirand JH, Fayad LE, Rodriguez AM, Romaguera JE, Hagemeister FB, et al. Follicular dendritic cell sarcoma: a report of 14 cases and a review of the literature. Am J Hematol. 2007;82(8):725728.

14. Azim HA, Elsedewy E, Azim HA, Jr. Imatinib in the treatment of follicular dendritic sarcoma: a case report and review of literature. Onkologie. 2007;30(7):381-384.

15. Li J, Duan MH, Han B, Zhang W, Zhou DB, Zhao YQ, Shen T. [Dendritic cell sarcoma: 4 cases report with literature review]. Zhonghua Xue Ye Xue Za Zhi. 2008;29(4):263-266.

16. Choi BS, Baek JH, Shin YM, Kim JH, Kim HW, Lee SJ, Cha HJ. Follicular dendritic cell sarcoma: a case report and review of the literature. Cancer Res Treat. 2010;42(2):121-124.

17. Cyriac S, Praveenkumar D, Majhi U, Sagar TG. Follicular dendritic cell sarcoma of the neck with an aggressive and fatal course. J Cancer Res Ther. 2010;6(1):114-116.

18. Shinagare AB, Ramaiya NH, Jagannathan JP, Hornick JL, Swanson RS. Primary follicular dendritic cell sarcoma of liver treated with cyclophosphamide, doxorubicin, vincristine, and prednisone regimen and surgery. J Clin Oncol. 2011;29(35):e849-851.

19. Pizzi M, Ludwig K, Palazzolo G, Busatto G, Rettore C, Altavilla G. Cervical follicular dendritic cell sarcoma: a case report and review of the literature. Int J Immunopathol Pharmacol. 2011;24(2):539-544.

20. Bushan K. Follicular Dendritic Cell Sarcoma Mediastinum - a case report. Indian J Surg Oncol. 2014;5(4):290292.

21. Dimri K, Trehan R, Pandey AK, Khosla D. Long term survival after whole brain radiotherapy for brain metastasis in follicular dendritic cell sarcoma. Indian J Cancer. 2014;51(3):395-396.

22. Conry RM. Response of follicular dendritic cell sarcoma to gemcitabine and docetaxel: report of two cases and literature review. Clin Sarcoma Res. 2014;4:6.

23. Purkait S, Mallick S, Joshi PP, Mallick S, Murugan NV, Sharma MC, Suri V, et al. Retroperitoneal and mediastinal follicular dendritic cell sarcoma: report of 3 cases with review of literature. Hematol Oncol. 2017;35(3):374-379.

24. Ma Y, Sun J, Yang C, Yuan D, Liu J. Follicular dendritic cell sarcoma: two rare cases and a brief review of the literature. Onco Targets Ther. 2015;8:1823-1830.

25. Gu LI, Ouyang C, Lu F. Follicular dendritic cell sarcoma with extensive lymph node involvement: A case report. Oncol Lett. 2015;10(1):399-401.

26. Sahu KK, Prakash G, Rao S, Bal A, Malhotra P, Ahluwalia J, Vashistha RK. A rare case of follicular dendritic cell sarcoma with pseudochylous effusion and review of literature from India. Indian J Hematol Blood Transfus. 2015;31(2):307-310.

27. Liberale G, Keriakos K, Azerad M, De Saint Aubain N, El Nakadi I. Intraperitoneal follicular dendritic cell sarcoma: role of chemotherapy and bone marrow allotransplantation in locally advanced disease? Clin Med Insights Oncol. 2015;9:9-13. 
28. Spatola C, Migliore M, Emanuele Liardo RL, Bevilacqua R, Luigi R, Vincenzo S, Tocco A, et al. Follicular dendritic cell sarcoma of mediastinum: a key role of radiotherapy in a multidisciplinary approach. Future Oncol. 2015;11(24 Suppl):57-61.

29. Lemech CR, Williams R, Thompson SR, McCaughan B, Chin M. Treatment of breast cancer 2 (BRCA2)-mutant follicular dendritic cell sarcoma with a poly ADP-ribose polymerase (PARP) inhibitor: a case report. BMC Res Notes. 2016;9:386.

30. Sasaki M, Izumi H, Yokoyama T, Kojima M, Hosono A. Follicular dendritic cell sarcoma treated with a variety of chemotherapy. Hematol Oncol. 2017;35(4):905-908.

31. Bouriga R, Abdessaied N, Hochlef M, Mallat N, Mahjoub M, Sriha B, Ahmed SB. Retroperitoneal follicular dendritic cell sarcoma in a young woman: Diagnosis and treatment challenges. Curr Probl Cancer. 2018;42(3):350357.

32. Chen HM, Shen YL, Liu M. Primary hepatic follicular dendritic cell sarcoma: A case report. World J Clin Cases. 2019;7(6):785-791.

33. Li L, Shi YH, Guo ZJ, Qiu T, Guo L, Yang HY, Zhang $\mathrm{X}$, et al. Clinicopathological features and prognosis assessment of extranodal follicular dendritic cell sarcoma. World J Gastroenterol. 2010;16(20):2504-2519.

34. Pang J, Mydlarz WK, Gooi Z, Waters KM, Bishop J, Sciubba JJ, Kim YJ, et al. Follicular dendritic cell sarcoma of the head and neck: Case report, literature review, and pooled analysis of 97 cases. Head Neck. 2016;38(Suppl 1):E2241-2249. 